\title{
Telocytes as potential targets in a cyclophosphamide-induced animal model of premature ovarian failure
}

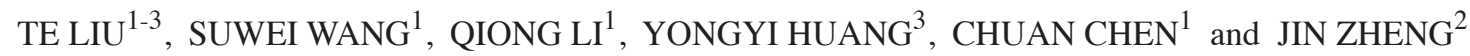 \\ ${ }^{1}$ Central Laboratory, Shanghai Geriatric Institute of Chinese Medicine; ${ }^{2}$ Department of Gynecological Traditional Chinese Medicine, \\ Longhua Hospital, Shanghai University of Traditional Chinese Medicine, Shanghai 200031; ${ }^{3}$ Central Laboratory, \\ Shanghai Tenth People's Hospital, Tongji University, Shanghai 200072, P.R. China
}

Received September 8, 2015; Accepted June 3, 2016

DOI: $10.3892 / \mathrm{mmr} .2016 .5540$

\begin{abstract}
Premature ovarian failure (POF) refers to the presence of ovarian atrophic permanent amenorrhea in women under the age of 40 . The pathogenesis of POF remains to be fully elucidated. Telocytes are a group of specialized cells with a small cell volume and very long cytoplasmic prolongations with dichotomous branching. Previous studies have indicated that telocytes function to support the trachea and serve as stem cell niches. Although it has been confirmed that telocytes are present in numerous organs in mammals, it remains to be determined whether they are present in ovarian tissues and whether they are involved in the development of POF. The present study used a cyclophosphamide-induced mouse model of POF. Hematoxylin and eosin staining and an enzyme-linked immunosorbent assay revealed that cyclophosphamide induced edema and apoptosis of ovarian stromal and granulosa cells and increased atretic follicles. In addition, cyclophosphamide induced abnormal peripheral blood FSH and E2 levels in mice. Transmission electron microscopy revealed a small number of telocyte-like cell structures in the ovarian stroma of wild-type mice. In addition, flow cytometry and immunohistochemical staining results suggested that the number of cluster of differentiation (CD)34/platelet-derived growth factor receptor (PDGFR) $\alpha$, CD34/PDGFR $\beta$ and CD34/vimentin double-positive cells in the ovaries of POF mice was significantly decreased compared with wild-type mice. In conclusion, mouse ovarian tissues appear to contain
\end{abstract}

Correspondence to: Professor Jin Zheng, Department of Gynecological Traditional Chinese Medicine, Longhua Hospital, Shanghai University of Traditional Chinese Medicine, 725 Wanping Road, Shanghai 200031, P.R. China

E-mail: jinzheng2013@126.com

Professor Chuan Chen, Central Laboratory, Shanghai Geriatric Institute of Chinese Medicine, Longhua Hospital, Shanghai University of Traditional Chinese Medicine, 365 Xiangyang Road, Shanghai 200031, P.R. China

E-mail: chuanchen9453@126.com

Key words: telocytes, cyclophosphamide, premature ovarian failure telocytes, and cyclophosphamide treatment significantly reduced the number of ovarian telocytes. Therefore, telocytes may serve as a potential novel marker of POF induced by cyclophosphamide.

\section{Introduction}

Premature ovarian failure (POF) refers to the presence of ovarian atrophy-associated permanent amenorrhea in women under $40(1,2)$. The levels of follicle stimulating hormone (FSH) are significantly increased in peripheral blood while the levels of estradiol (E2) are significantly decreased $(1,3)$. Histopathological examination of ovarian tissues has revealed significant atrophy and fibrosis, marked follicular atresia and the absence of normal follicular stages (including primordial follicle, antral follicle, cumulus oophorus and mature egg) (3-5). The underlying mechanisms of POF development are diverse and complex. Currently, the pathogenesis of POF remains to be elucidated, and no effective treatment measures exist.

Telocytes are a group of highly specialized cells in the connective tissues of organs (6-8). Telocytes have a small cell volume and long cytoplasmic prolongations (typically $10-100 \mu \mathrm{m}$ in length), referred to as telopodes (9-11). The dichotomous branching structure of telopodes may be observed by electron microscopy (12-14), which reveals that telopodes consist of thick branching segments (podoms) and thin branching segments (podomers) $(15,16)$. It has been demonstrated that telocytes are present in the connective tissues of numerous organs in mammals, including myocardial tissue $(6,9,11,12)$, uterus and fallopian tubes $(10,11,16)$, placental tissues $(11)$, ureter $(10,11,17)$, skeletal muscle $(11,17,18)$, lung $(9,11)$, mammary gland (11), liver $(11,19-21)$ and pancreas $(11,14)$. Although no telocyte-specific marker has been identified to date, previous studies have reported increased expression of cluster of differentiation (CD)34, CD117 (c-kit), platelet-derived growth factor receptor (PDGFR) $\alpha$, PDGFR $\beta$ and vimentin (6-22). Previous studies have demonstrated that telocytes have the following biological functions: i) Telocytes may guide the migration of cardiac stromal cells from the endothelium to mesothelium and contribute to vascular regeneration of myocardial tissues following myocardial infarction $(6,8,11,12)$; ii) telocytes form a complex network 
structure in the lung via their telopodes that support the bronchial lumens of lobules, thus preventing obstruction of lobules and small blood vessels with weak vascular walls during the respiratory process $(9,12)$; iii) telocytes affect antigen presentation, immune activation and immune tolerance, via the release of microvesicles during synapses with immune cells (8); and iv) as 'stem cell helper cells', telocytes facilitate repair and regeneration at injured sites through cross-linking to form stem cell niches $(11,12,16,20)$. However, whether telocytes are present in the mammalian ovary and contribute to the regulation of ovarian function remains to be determined (11). In the present study, electron microscopy combined with immunofluorescence analysis and flow cytometry was performed to investigate the hypothesis that telocytes are present in ovarian tissues in mice. In addition, telocytes were investigated as a potential novel marker of POF in mice induced by cyclophosphamide.

\section{Materials and methods}

Establishment of a POF mouse model. POF was induced in mice according to a previously described method (3-5). Briefly, 8 -week-old female C57BL/6 mice $(n=40)$ were purchased from the Experimental Animal Center of Shanghai University of Traditional Chinese Medicine (Shanghai, China). Mice were housed in a temperature-controlled environment under standard light-dark cycles with ad libitum access to food and water as previously described (3). Mice were randomly divided into two groups (20 mice/group). Mice in the POF group were injected intraperitoneally with $70 \mathrm{mg} / \mathrm{kg}$ cyclophosphamide (Sigma-Aldrich, St. Louis, MO, USA), followed by intraperitoneal injections of $30 \mathrm{mg} / \mathrm{kg}$ cyclophosphamide every three days for three weeks, to establish the POF mouse model. Mice in the control group were injected intraperitoneally with an equal volume of normal saline every three days for three weeks. All mice were sacrificed by cervical dislocation at three weeks and the ovaries were harvested for subsequent analysis. The study was approved by the Ethics Committee of Shanghai Geriatric Institute of Chinese Medicine (Shanghai, China; ref. SHAGESYDW2015026). All experiments conformed to standards set by the Laboratory Animal Regulation of the State Scientific and Technological Commission (www.slarc.org.cn).

Hematoxylin and eosin $(H \& E)$ staining. $\mathrm{H} \& \mathrm{E}$ staining was performed according to a previously described method (3). Briefly, fresh tissues were immersed in $4 \%$ paraformaldehyde (Sigma-Aldrich) for $30 \mathrm{~min}$ at room temperature to allow fixation. Tissues were dehydrated using an ethanol gradient, embedded in paraffin, sectioned (thickness, $6 \mu \mathrm{m}$ ) and deparaffinized in xylene (Sigma-Aldrich). Tissue sections were stained with H\&E (Sigma-Aldrich), cleared in xylene and mounted on slides using neutral balsam (Sigma-Aldrich). The number of atretic follicles was counted per microscope field, in three non-overlapping fields of ovary sections from each mouse.

Immunofluorescence staining. Immunofluorescence staining was performed as previously described (4). Briefly, fresh tissues were fixed and sectioned as for H\&E staining. Tissue sections were incubated with immunofluorescence blocking solution (Beyotime Institute of Biotechnology, Haimen, China) for $30 \mathrm{~min}$ at $37^{\circ} \mathrm{C}$. Subsequently, sections were washed three times in immunofluorescence wash solution (Beyotime Institute of Biotechnology) for $5 \mathrm{~min}$ at room temperature. Sections were incubated with primary antibodies (Table I) for $45 \mathrm{~min}$ at $37^{\circ} \mathrm{C}$. The washing step was repeated, and sections were incubated with secondary antibodies (Table II) for $45 \mathrm{~min}$ at $37^{\circ} \mathrm{C}$. Following a final repeat of the washing step, sections were mounted using Kisser's Mounting Medium (Beyotime Institute of Biotechnology).

Immunohistochemical staining. Immunohistochemical staining was performed as previously described (4). Briefly, ovarian tissue samples were washed three times with phosphate-buffered saline (PBS; Sigma-Aldrich), fixed with 4\% paraformaldehyde (Sigma-Aldrich) for $30 \mathrm{~min}$, dehydrated through a graded series of ethanol, cleared in xylene and embedded in paraffin. Subsequently, serial 6- $\mu$ m thick sections were cut, rinsed with 3\% phosphate buffer (Sigma-Aldrich), and subjected to heat retrieval in a microwave. The sections were then incubated with primary antibodies (Table I) for $45 \mathrm{~min}$ at $37^{\circ} \mathrm{C}$. Subsequently, sections were washed three times in immunohistochemistry wash solution (Beyotime Institute of Biotechnology) for $5 \mathrm{~min}$ at room temperature. Sections were incubated with secondary antibodies (Table II) for $45 \mathrm{~min}$ at $37^{\circ} \mathrm{C}$. Following a repeat of the washing step, ABC chromogenic reagent (Sigma-Aldrich) was added to visualize bound antibody. PBS (pH 7.4) was used as a negative control for primary antibody. Sections were mounted using neutral balsam (Beyotime Institute of Biotechnology). Five randomly selected fields of view (magnification, x200; Olympus BX43; Olympus Corporation, Tokyo, Japan) were assessed for each tissue section and analyzed using Integrated Performance Primitives software version 4.0 (Intel Corporation, Santa Clara, CA, USA).

Enzyme-linked immunosorbent assay (ELISA). Mouse blood was obtained by retro-orbital blood collection at sacrifice ( $\sim 100 \mu$ l blood from each mouse) and centrifuged at $453 \mathrm{x} \mathrm{g}$ at $4^{\circ} \mathrm{C}$ for $10 \mathrm{~min}$. The supernatants were collected and these serum samples were used for ELISA. ELISAs were performed according to the manufacturer's instructions for the mouse E2 and FSH ELISA kits (Westang Bio-Tech Co., Ltd., Shanghai, China). Briefly, $100 \mu \mathrm{l}$ serum, along with mouse E2 standards (at concentrations of 8,000, 4,000, 2,000, 1,000, 500, 250 and $125 \mathrm{pg} / \mathrm{ml}$ ) and mouse FSH standards (at concentrations of $10,5,2.5,1.25,0.625,0.312$ and $0.156 \mathrm{ng} / \mathrm{ml})$, was added to pre-coated 96-well detection plates and incubated for $60 \mathrm{~min}$ at $37^{\circ} \mathrm{C}$. The solution was discarded and the plates were washed three times with wash solution. Horseradish peroxidase-conjugated detection antibodies were added and incubated for $60 \mathrm{~min}$ at $37^{\circ} \mathrm{C}$. Subsequently, stop solution was added and the optical density of each well in the 96-well plate was measured at a wavelength of $450 \mathrm{~nm}$.

Flow cytometry. Cells were isolated and flow cytometry was performed according to a previously described method $(5,10)$. Briefly, the murine ovaries were minced into small pieces $\left(\sim 1 \mathrm{~mm}^{3}\right)$ and washed three times with $\mathrm{PBS}$ at $4^{\circ} \mathrm{C}$. The samples were digested using $10 \mathrm{mg} / \mathrm{ml}$ collagenase type I 
Table I. Primary antibodies.

\begin{tabular}{|c|c|c|c|c|c|c|}
\hline Target & Species raised in & Species raised against & Application & Dilution & Catalog no. & Supplier \\
\hline Inhibin $\alpha$ & Rabbit & Mouse & IHC & $1: 100$ & sc-30146 & $\mathrm{SC}$ \\
\hline Inhibin $\beta$ & Rabbit & Mouse & IHC & $1: 100$ & sc-50288 & $\mathrm{SC}$ \\
\hline $\mathrm{AMH}$ & Rabbit & Mouse & IHC & $1: 100$ & ab103233 & $\mathrm{AB}$ \\
\hline FSHR & Rabbit & Mouse & IHC & $1: 100$ & sc-13935 & $\mathrm{SC}$ \\
\hline PDGFR $\alpha$ & Rabbit & Mouse & $\mathrm{IF}, \mathrm{FC}$ & $1: 100$ & sc-431 & $\mathrm{SC}$ \\
\hline PDGFR $\beta$ & Rabbit & Mouse & IF, FC & $1: 100$ & sc-432 & $\mathrm{SC}$ \\
\hline CD34 & Goat & Mouse & IF & $1: 100$ & sc-7045 & $\mathrm{SC}$ \\
\hline c-Kit & Rabbit & Mouse & IF, FC & $1: 100$ & sc-5535 & $\mathrm{SC}$ \\
\hline Vimentin & Rat & Mouse & IF, FC & $1: 100$ & sc-5565 & $\mathrm{SC}$ \\
\hline
\end{tabular}

AMH, anti-müllerian hormone; FSHR, follicle-stimulating hormone receptor; PDGFR, platelet-derived growth factor receptor; CD, cluster of differentiation; c-Kit, CD117; IHC, immunohistochemistry; IF, immunofluorescence; FC, flow cytometry; SC, Santa Cruz Biotechnology, Inc. (Dallas, TX, USA); AB, Abcam (Cambridge, MA, USA).

(Sigma-Aldrich) and $0.1 \%$ hyaluronidase (Sigma-Aldrich) in PBS for $5 \mathrm{~min}$ on an orbital shaker at $37^{\circ} \mathrm{C}$, and filtered through $40-\mu \mathrm{m}$ diameter cell strainers. These granulosa cells from ovaries were fixed in $4 \%$ paraformaldehyde for $30 \mathrm{~min}$ at room temperature. Following centrifugation at $453 \mathrm{x} g$ for $5 \mathrm{~min}$ at $37^{\circ} \mathrm{C}$, the supernatant was discarded and the cell precipitate was resuspended in blocking solution (Beyotime Institute of Biotechnology) for $30 \mathrm{~min}$ at $37^{\circ} \mathrm{C}$. Cells were centrifuged, the blocking solution was discarded, and primary antibodies (Table I) were added and incubated for $45 \mathrm{~min}$ at $37^{\circ} \mathrm{C}$. Following centrifugation, the antibodies were discarded and wash solution (Beyotime Institute of Biotechnology) was added to the cells for $5 \mathrm{~min}$ at room temperature. Cells were then centrifuged, and secondary antibodies (Table II) were added and incubated for $30 \mathrm{~min}$ at $37^{\circ} \mathrm{C}$. Following centrifugation, the antibodies were discarded and wash solution (Beyotime Institute of Biotechnology) was added to the cells for $5 \mathrm{~min}$ at room temperature. Positive cells were detected by flow cytometry (Quanta SC; Beckman Coulter, Inc., Brea, CA, USA).

Preparation of samples and observation using electron microscopy. Samples were fixed and embedded according to a previous study (23) and the protocol provided by KingMed Center for Clinical Laboratory Co., Ltd. (Guangzhou, China). Tissue samples were fixed in $1 \%$ glutaraldehyde (Sigma-Aldrich) for $4 \mathrm{~h}$ followed by $1 \%$ osmium tetroxide (Sigma-Aldrich) for $1 \mathrm{~h}$. Samples were then dehydrated in acetone and embedded in Eponate 12 resin (Ted Pella, Inc., Redding, CA, USA). Samples were cut into ultrathin sections (thickness, $70 \mathrm{~nm}$ ), placed on copper grids and stained using $1 \%$ uranyl acetate and $1 \%$ lead citrate (Sigma-Aldrich). The sections were observed and images captured using a JEM-1230 transmission electron microscope (JEOL, Ltd., Tokyo, Japan).

Statistical analysis. Statistical analyses were performed in GraphPad Prism software version 5.00 (GraphPad Software, Inc., La Jolla, CA, USA). The data are presented as the mean \pm standard error where applicable; differences were evaluated using Student's $t$-test. $\mathrm{P}<0.05$ was considered to indicate a statistically significant difference.

\section{Results}

Cyclophosphamide induces ovarian damage and abnormal hormone secretion in mice. Histological analysis with $\mathrm{H} \& \mathrm{E}$ staining revealed that the number of atretic follicles in the ovaries of cyclophosphamide-treated mice was significantly increased compared with the control group ( $\mathrm{P}=0.018$; Fig. 1A). In addition, the texture of ovarian tissues was dense in control mice. Various stages of follicles were observed in ovaries, including primordial follicles (Fig. 1B; pink arrows), antral follicles (Fig. 1B; red arrows), cumulus oophorus (Fig. 1B; blue arrows) and mature eggs (Fig. 1B; black arrows). Following treatment with cyclophosphamide for three weeks, ovarian tissues demonstrated significant atrophy, the ovarian stroma had empty space, and apoptosis and necrosis in ovarian granulosa cells was significant (Fig. 1B; yellow arrows). In addition, it was difficult to identify the various stages of follicles and mature eggs, and atretic follicles and luteal tissue atrophy were abundant (Fig. 1B). Immunohistochemistry results (Fig. 1C) indicated that the expression of the ovarian granulosa cell markers anti-Müllerian hormone (AMH) and inhibin $\alpha / \beta$ in the ovaries of POF mice was reduced compared with control mice; however, no difference was observed in FSH receptor (FSHR) staining between the two groups. The weight of ovarian tissues from cyclophosphamide-treated mice was significantly reduced compared with that of control mice $(\mathrm{P}=0.022$; Fig. 2A). ELISA results demonstrated that the peripheral blood $\mathrm{E} 2$ level in mice treated with cyclophosphamide was significantly reduced compared with the control group $(\mathrm{P}=0.015$; Fig. $2 \mathrm{~B})$, and the FSH level was significantly increased compared with the control group ( $\mathrm{P}=0.027$; Fig. 2C). Histological and hormone level detection results therefore suggested that cyclophosphamide induced ovarian granulosa cell injury and apoptosis as well as the development of POF.

Telocytes are present in normal ovarian tissues. Transmission electron microscopy was performed to confirm that telocytes 
Table II. Secondary antibodies.

\begin{tabular}{lcccccc}
\hline Species raised in & Species raised against & Conjugated to & Application & Dilution & Catalog no. & Supplier \\
\hline Donkey & Goat & Cy3 & IF, FC & $1: 100$ & ab6949 & AB \\
Goat & Rabbit & FITC & IF, FC & $1: 100$ & sc-2777 & SC \\
Goat & Rat & FITC & IF, FC & $1: 100$ & sc-2011 & SC \\
Donkey & Rabbit & HRP & IHC & $1: 300$ & ab205722 & AB \\
\hline
\end{tabular}

Cy3, cyanine 3; FITC, fluorescein isothiocyanate; HRP, horseradish peroxidase; IHC, immunohistochemistry; IF, immunofluorescence; FC, flow cytometry; AB, Abcam (Cambridge, MA, USA); SC, Santa Cruz Biotechnology, Inc. (Dallas, TX, USA).

A
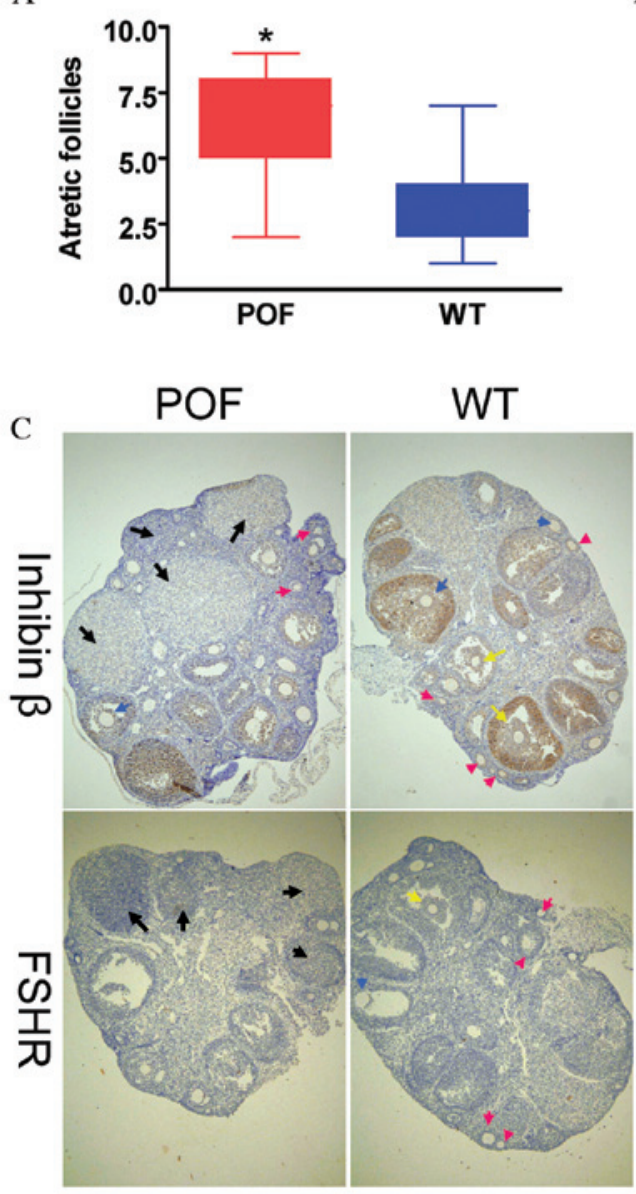
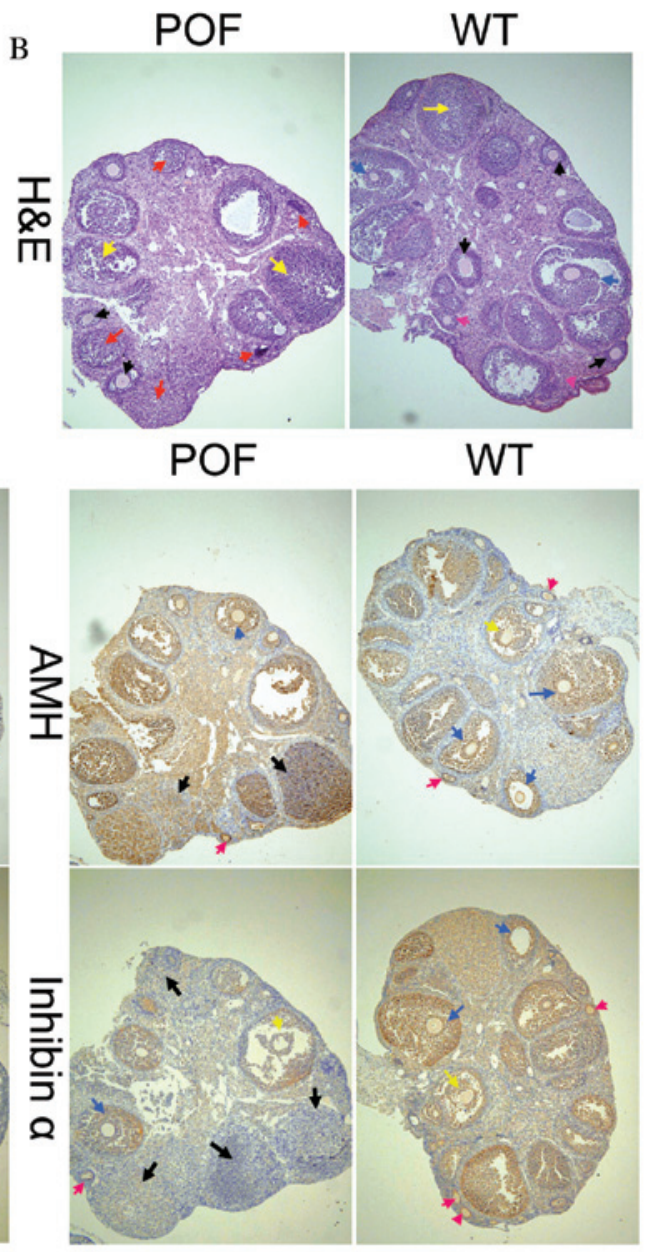

Figure 1. Histological analysis of ovaries. (A) The number of atretic follicles in the ovarian tissues of POF mice was significantly increased compared with control mice. ${ }^{*} \mathrm{P}<0.05$ vs. WT; $\mathrm{n}=10$. (B) H\&E staining of ovarian tissues. Granulosa cells in the ovarian tissues of POF mice demonstrated clear edema and apoptosis, and increased atretic follicles. Ovarian granulosa cell injury is indicated by yellow arrows, primordial follicles by pink arrows, antral follicles by red arrows, mature oocytes by black arrows and cumulus oophorus by blue arrows. (C) Immunohistochemical staining revealed that in ovarian tissues of POF mice, the expression of the ovarian granulosa cell markers AMH, inhibin $\alpha$ and inhibin $\beta$ was reduced compared with those of control mice. No difference was observed in the expression of FSHR between the two groups. Black arrows indicate atretic follicles, pink arrows indicate primordial follicles, yellow arrows indicate cumulus oophorus and blue arrows indicate mature oocytes. Magnification, x100. H\&E, hematoxylin and eosin; POF, premature ovarian failure; WT, wild-type; AMH, anti-Müllerian hormone; FSHR, follicle stimulating hormone receptor.

were present in ovarian tissues. A unique type of cell body was observed in the ovarian stroma in control mice. Although the cell volume was small, it had very long telopodes, which had a typical dichotomous branching structure and alternating thick and thin segments (Fig. 3). However, cells with these morphological features were not observed in ovarian tissues from POF mice. Following a review of the literature, it was hypothesized that these cells were telocytes.

Cyclophosphamide induces a reduction in telocyte number and marker expression levels. Flow cytometry detection of telocyte markers revealed that the number of CD34/PDGFR $\alpha$, 

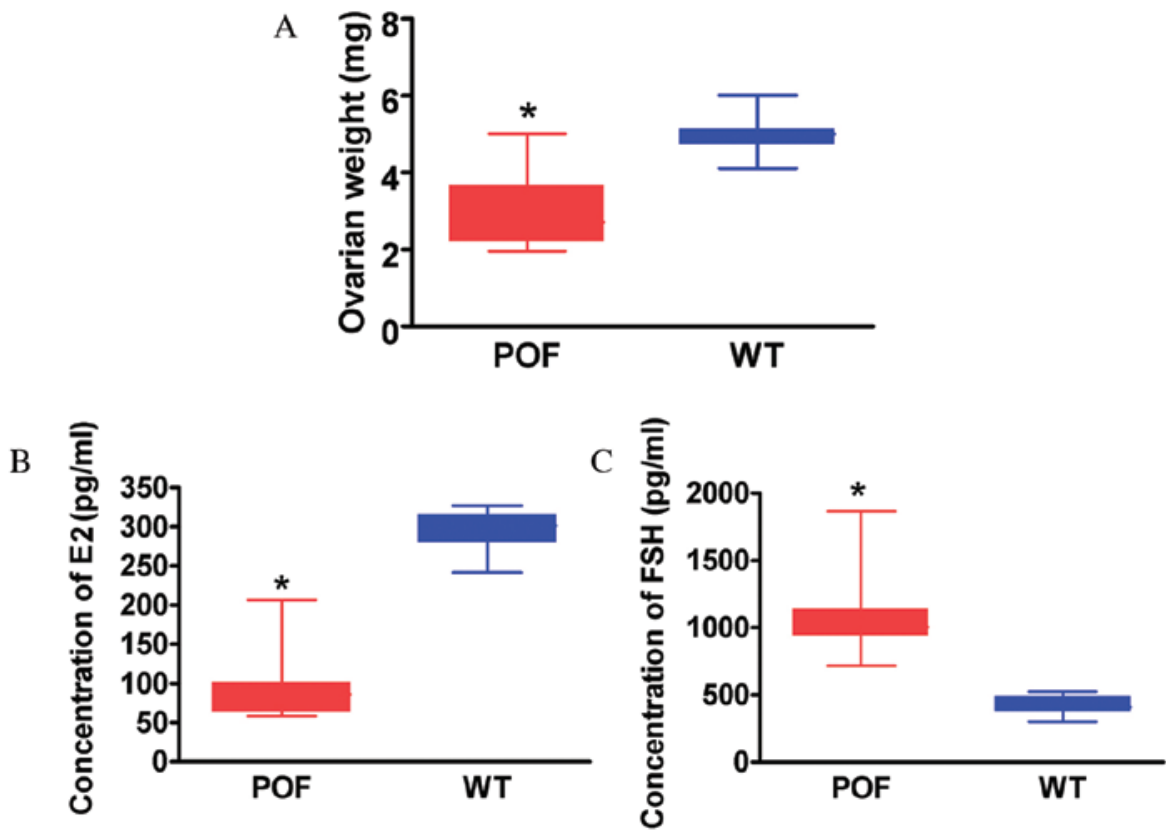

Figure 2. Characteristics of POF mice. (A) The weight of ovarian tissues from POF mice was significantly reduced compared with control mice. (B) The peripheral blood E2 level in POF mice was significantly reduced compared with control mice. (C) The peripheral blood FSH level in POF mice was significantly increased compared with control mice. "P<0.05 vs. WT; $n=20$. POF, premature ovarian failure; WT, wild-type; E2, estradiol; FSH, follicle stimulating hormone.

CD34/PDGFR $\beta$ and CD34/vimentin double-positive cells in the ovaries of POF mice was significantly reduced compared with control mice $(\mathrm{P}=0.031$; Fig. 4$)$, whereas the number of CD34/c-kit double-positive cells was not significantly different between the two groups ( $\mathrm{P}=0.061$; Fig. 4). In addition, immunofluorescence staining results indicated that the number of CD34/PDGFR $\alpha$, CD34/PDGFR $\beta$ and CD34/vimentin double-positive cells in the ovarian tissues of POF mice was decreased compared with control mice (Fig. 5). These results suggested that the number of telocytes significantly decreased in ovarian tissues of mice following cyclophosphamide treatment.

\section{Discussion}

POF is associated with complex risk factors and high morbidity (2). Typically, studies have focused on the effects of risk factors, including genetic variations, immunity and inflammation, environmental hormones and contamination, personal stress, and endocrine disorders (1,2). During the development of $\mathrm{POF}$, lesions are most prominent in egg and ovarian granulosa cells $(4,5,24)$. Ovarian granulosa cells, similar to Sertoli cells of the male reproduction system, support female reproductive cells i.e. mature egg cells (3), surrounding these cells during follicle development to form a unique microenvironment. Ovarian granulosa cells secrete hormones and cytokines for egg maturation, including estrogen, FSH, anti-Müllerian antibody, inhibin $\alpha / \beta$ and growth hormone, to support the development, maturation and ovulation of follicles (3). Ovarian granulosa cell apoptosis reduces the follicular reserve capacity of the ovary, thus causing the development of POF $(1,5)$. In the ovaries of POF patients, ovarian granulosa cells typically exhibit apoptosis and edema (4). The primary function of the

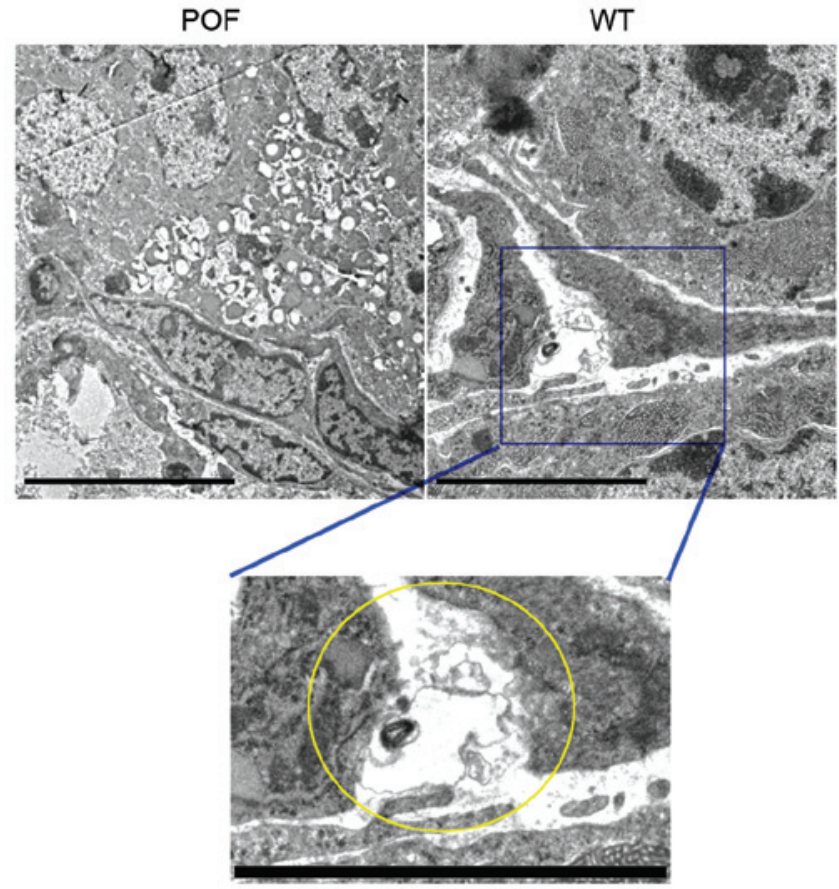

Figure 3. Transmission electron microscopy of ovarian tissues. Telocyte-like cells were observed in the stroma of ovarian tissues in mice of the WT group; these cells had a triangular cell body and telopode-like prolongations with a dichotomous branching structure. However, cells with these morphological features were not observed in the ovaries of POF mice. The yellow circle indicates a telocyte-like cell in the magnification of the blue square from the WT image. Scale bar $=5 \mu \mathrm{m}$; magnification, $\mathrm{x} 5,000$. POF, premature ovarian failure; WT, wild-type.

ovary is to provide a location for egg maturation. Therefore, in the ovaries of POF patients, the number of atretic follicles 


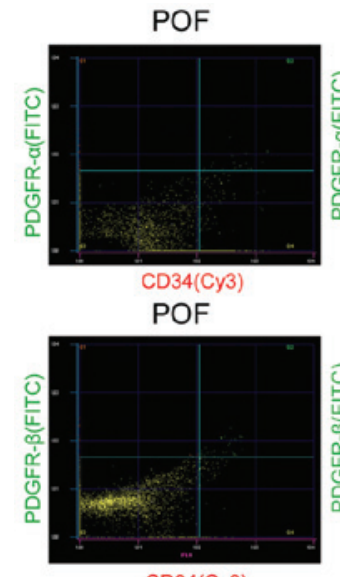

$\mathrm{CD} 34(\mathrm{Cy} 3)$

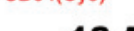

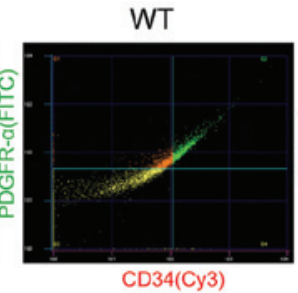

WT

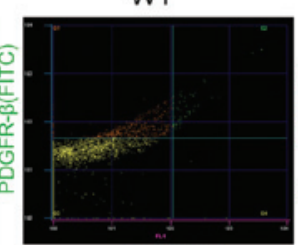

CD34(Cy3)

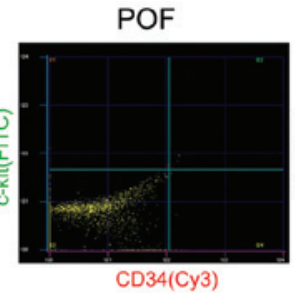

POF

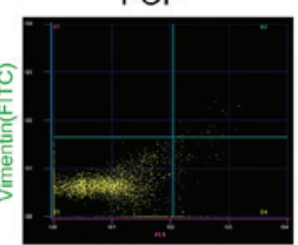

CD34(Cy3)

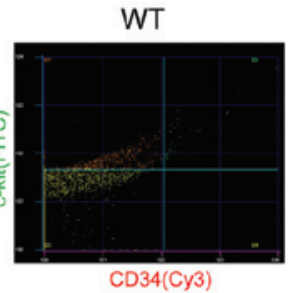

WT

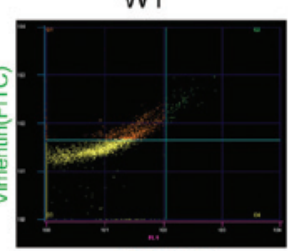

CD34(Cy3)
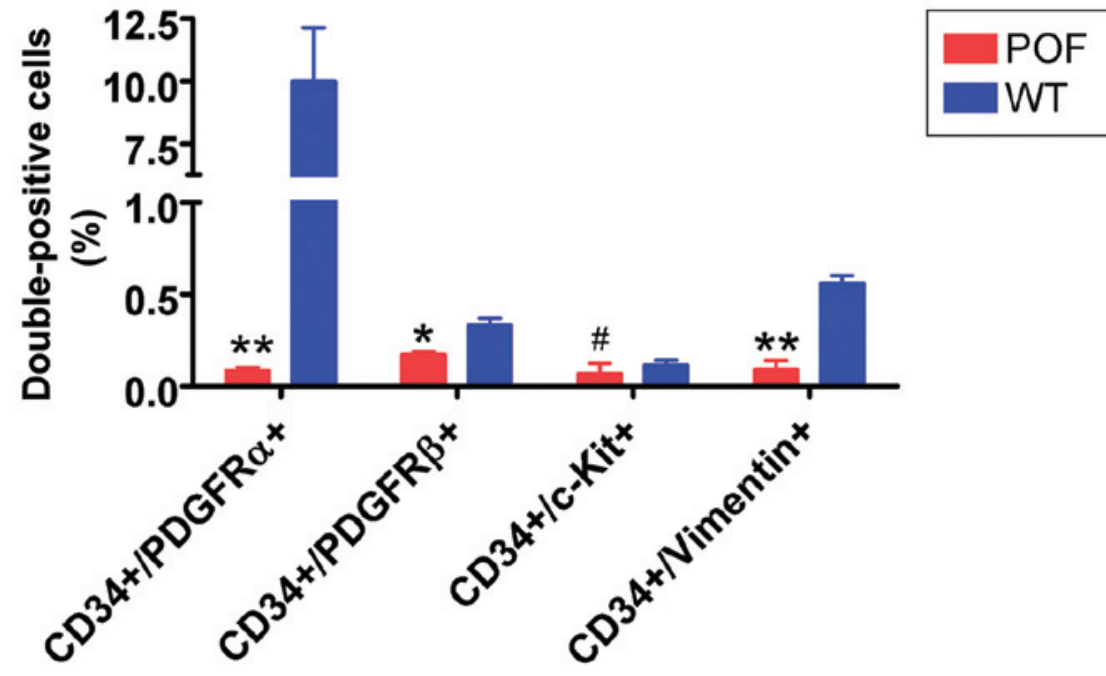

Figure 4. Detection of the expression levels of telocyte markers in ovarian tissues by flow cytometry. The number of CD34/PDGFR $\alpha$, CD34/PDGFR $\beta$ and $\mathrm{CD} 34 /$ vimentin double-positive cells in ovarian cells in WT mice was significantly increased compared with $\mathrm{POF}$ mice. ${ }^{\sharp} \mathrm{P}>0.05$, ${ }^{*} \mathrm{P}<0.05$ and ${ }^{* *} \mathrm{P}<0.01$ vs. WT; $n=10$. POF, premature ovarian failure; WT, wild-type; CD, cluster of differentiation; PDGFR, platelet-derived growth factor receptor; c-kit, CD117; FITC, fluorescein isothiocyanate; Cy3, cyanine 3 .

is significantly increased and the number of healthy follicles is significantly decreased; in addition, the number of follicles at various stages is significantly decreased (2). As well as egg and ovarian granulosa cells, other types of cell, including ovarian stromal cells, ovarian epithelial cells and ovarian microvascular endothelial cells are affected by POF; however, there have been limited studies on their functions.

A literature search revealed reports of a unique cell type present in the respiratory, cardiovascular and digestive systems; these cells were telocytes (6-22). Telocytes are predominantly localized in the connective tissues of organs. Telocyte features include a small cell volume, very long cytoplasmic prolongations (telopodes) and a typical dichotomous branching structure of these telopodes (6-22). Telopodes comprise a complex network structure, which supports the lumen of lobular bronchioles and blood vessels to prevent obstruction. In addition, as 'stem cell helper cells', telocytes form stem cell niches through cross-linking to facilitate the repair and regeneration of injured sites (6-22). However, in mammals, not every organ contains telocytes and it has not been reported whether they are present in ovaries.

As telocytes function to maintain the microenvironment, it was hypothesized that ovaries may contain telocytes.
Therefore, transmission electron microscopy was performed to analyze the ultra-microstructure of ovarian tissues from control and POF mice. Very long cells were observed in the stroma of healthy mouse ovaries. The somatic synapses of these cells revealed a triangular shape and very long and thin prolongations with a typical dichotomous branching structure, suggesting that these cells may be ovarian tissue telocytes. However, cells with the above morphological features were not observed in the ovaries of POF mice. Subsequently, the expression levels of the telocyte-specific markers CD34, CD117, PDGFR $\alpha / \beta$ and vimentin were evaluated in ovarian tissues. The results of flow cytometry and immunofluorescence suggested that the number of CD34/PDGFR $\alpha, \mathrm{CD} 34 / \mathrm{PDGFR} \beta$ and CD34/vimentin double-positive cells in ovarian cells of wild-type mice was significantly increased compared with POF mice. In addition, immunohistochemical staining and flow cytometry experiments suggested that various telocyte markers were expressed in the ovarian tissues of control mice. However, when mice were treated with cyclophosphamide, the expression of telocytes and their markers decreased. Based on the results of the present study, telocytes in ovarian tissues may have an important function in maintaining the ovarian microenvironment. The ovarian tissue injury induced by 


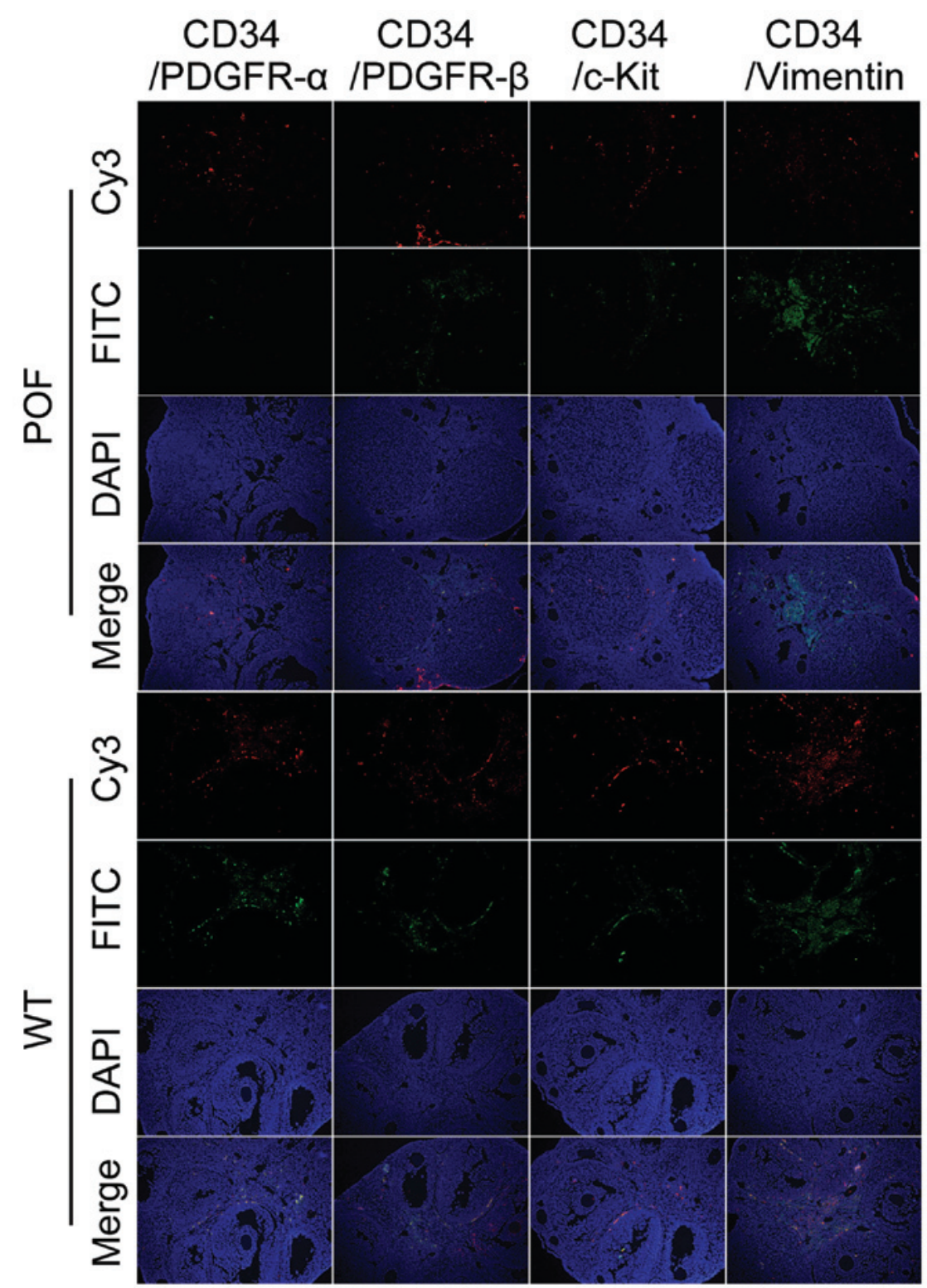

Figure 5. Detection of the expression of telocyte markers in ovarian tissues by immunohistochemistry. The number of CD34/PDGFR $\alpha$, CD34/PDGFR $\beta$ and CD34/vimentin double-positive cells in ovarian tissues from POF mice was decreased compared with WT mice. POF, premature ovarian failure; WT, wild-type; CD, cluster of differentiation; PDGFR, platelet-derived growth factor receptor; c-kit, CD117; FITC, fluorescein isothiocyanate; Cy3, cyanine 3.

cyclophosphamide was not confined to ovarian granulosa cells and eggs, as ovarian telocytes were also affected.

In conclusion, mouse ovarian tissues appear to contain telocytes, which highly express CD34, PDGFR $\alpha / \beta$ and vimentin. The numbers of these cells were significantly reduced following cyclophosphamide treatment. Therefore, telocytes may serve as a potential novel marker of POF induced by cyclophosphamide. Future studies are required to investigate the mechanism underlying the effect of POF on telocytes.

\section{Acknowledgements}

The present study was supported by the National Natural Science Foundation of China (grant nos. 81273794 and 81202811), the China Postdoctoral Science Foundation (grant nos. 2014M550250 and 2015T80455) and the Shanghai Natural Science Foundation (grant no. 16ZR1434000).

\section{References}

1. Beck-Peccoz P and Persani L: Premature ovarian failure. Orphanet J Rare Dis 1: 9, 2006

2. Vujović S, Ivović M, Tancić-Gajić M, Marina L, Barać M, Arizanović Z, Nenezić A, Ivanisević M, Micić J, Sajić S and Micić D: Premature ovarian failure. Srp Arh Celok Lek 140: 806-811, 2012.

3. Liu T, Huang Y, Zhang J, Qin W, Chi H, Chen J, Yu Z and Chen C Transplantation of human menstrual blood stem cells to treat premature ovarian failure in mouse model. Stem Cells Dev 23: 1548-1557, 2014.

4. Liu T, Qin W, Huang Y, Zhao Y and Wang J: Induction of estrogen-sensitive epithelial cells derived from human-induced pluripotent stem cells to repair ovarian function in a chemotherapy-induced mouse model of premature ovarian failure. DNA Cell Biol 32: 685-698, 2013.

5. Liu T,Huang Y, Guo L, Cheng W and Zou G: CD44+/CD105+human amniotic fluid mesenchymal stem cells survive and proliferate in the ovary long-term in a mouse model of chemotherapy-induced premature ovarian failure. Int J Med Sci 9: 592-602, 2012.

6. Li H, Lu S, Liu H, Ge J and Zhang H: Scanning electron microscope evidence of telocytes in vasculature. J Cell Mol Med 18: 1486-1489, 2014. 
7. Hatta K, Huang ML, Weisel RD and Li RK: Culture of rat endometrial telocytes. J Cell Mol Med 16: 1392-1396, 2012.

8. Bei Y, Zhou Q, Fu S, Lv D, Chen P, Chen Y, Wang F and Xiao J: Cardiac telocytes and fibroblasts in primary culture: Different morphologies and immunophenotypes. PLoS One 10: e0115991, 2015.

9. Zheng Y, Cretoiu D, Yan G, Cretoiu SM, Popescu LM, Fang H and Wang X: Protein profiling of human lung telocytes and microvascular endothelial cells using iTRAQ quantitative proteomics. J Cell Mol Med 18: 1035-1059, 2014.

10. Li L, Lin M, Wang R, Zhang C, Qi G, Xu M, Rong R and Zhu T: Renal telocytes contribute to the repair of ischemically injured renal tubules. J Cell Mol Med 18: 1144-1156, 2014

11. Cretoiu SM and Popescu LM: Telocytes revisited. Biomol Concepts 5: 353-369, 2014.

12. Popescu LM, Curici A, Wang E, Zhang H, Hu S and Gherghiceanu M: Telocytes and putative stem cells in ageing human heart. J Cell Mol Med 19: 31-45, 2014.

13. Yang XJ, Yang J, Liu Z, Yang G and Shen ZJ: Telocytes damage in endometriosis-affected rat oviduct and potential impact on fertility. J Cell Mol Med 19: 452-462, 2014.

14. Vannucchi MG, Traini C, Manetti M, Ibba-Manneschi L and Faussone-Pellegrini MS: Telocytes express PDGFR $\alpha$ in the human gastrointestinal tract. J Cell Mol Med 17: 1099-1108, 2013.

15. Cismasiu VB and Popescu LM: Telocytes transfer extracellular vesicles loaded with microRNAs to stem cells. J Cell Mol Med 19: 351-358, 2015.
16. Roatesi I, Radu BM, Cretoiu D and Cretoiu SM: Uterine telocytes: A review of current knowledge. Biol Reprod 93: 10, 2015.

17. Vannucchi MG, Traini C, Guasti D, Del Popolo G and Faussone-Pellegrini MS: Telocytes subtypes in human urinary bladder. J Cell Mol Med 18: 2000-2008, 2014.

18. Li H, Zhang H, Yang L, Lu S and Ge J: Telocytes in mice bone marrow: Electron microscope evidence. J Cell Mol Med 18: 975-978, 2014

19. Fu S, Wang F, Cao Y, Huang Q, Xiao J, Yang C and Popescu LM: Telocytes in human liver fibrosis. J Cell Mol Med 19: 676-683, 2015.

20. Wang F, Song Y, Bei Y, Zhao Y, Xiao J and Yang C: Telocytes in liver regeneration: Possible roles. J Cell Mol Med 18: 1720-1726, 2014.

21. Li J, Shen F, Guan C, Wang W, Sun X, Fu X, Huang M, Jin J and Huang Z: Activation of Nrf2 protects against triptolide-induced hepatotoxicity. PLoS One 9: e100685, 2014.

22. Milia AF, Ruffo M, Manetti M, Rosa I, Conte D, Fazi M, Messerini L and Ibba-Manneschi L: Telocytes in Crohn's disease. J Cell Mol Med 17: 1525-1536, 2013.

23. Du X, Wang H, Xu F, Huang Y, Liu Z and Liu T: Enterovirus 71 induces apoptosis of SHSY5Y human neuroblastoma cells through stimulation of endogenous microRNA let-7b expression. Mol Med Rep 12: 953-959, 2015.

24. Hartmann BW, Kirchengast S, Albrecht A, Huber JC and Söregi G: Effect of hormone replacement therapy on growth hormone stimulation in women with premature ovarian failure. Fertil Steril 68: 103-107, 1997. 